\section{The therapeutic window of opportunity in rheumatoid arthritis: does it ever close?}

\author{
Karim Raza, ${ }^{1,2}$ Andrew Filer ${ }^{1,3}$
}

It is widely accepted in the rheumatology community that a 'therapeutic window of opportunity' exists for patients with rheumatoid arthritis (RA), that the disease is much more amenable to treatment within this window, and that treatment within the window can reset the disease's long-term trajectory in a way that treatment outside of it cannot. This concept is supported by data from studies which show that patients treated 'early', for example, within 3 months of the onset of symptoms, have favourable outcomes compared with patients treated later after symptom onset, ${ }^{1-4}$ and that aggressive therapy administered within an 'early' window can slow the rate of long-term structural damage. ${ }^{5}$ However, such data are equally consistent with a situation in which there is no point at which the 'window' closes but that earlier treatment is better than later treatment across the symptom duration spectrum. Understanding whether there is truly a time-limited window of opportunity in early RA is of critical importance. If such a window exists, then every effort must be made to see and treat patients before it closes and the research agenda needs to focus on the reasons for enhanced responsiveness within it.

Data from patients with RA from two large clinical cohorts have been analysed by van Nies et al to address the relationship between symptom duration at initial rheumatology assessment (a surrogate for the time from symptom onset to disease modifying anti-rheumatic drug (DMARD) commencement) and favourable outcomes, namely (1) DMARD-free sustained remission; (2) sustained remission irrespective of DMARD use and (3) low rates of radiological progression. ${ }^{6}$ Their key finding is that the relationships between symptom

${ }^{1}$ School of Immunity and Infection, College of Medical and Dental Sciences, University of Birmingham, Birmingham, UK; ${ }^{2}$ Department of Rheumatology, Sandwell and West Birmingham Hospitals NHS Trust, Birmingham, UK; ${ }^{3}$ Department of Rheumatology, University Hospitals Birmingham NHS Foundation Trust, Birmingham, UK

Correspondence to Professor Karim Raza, School of Immunity and Infection, College of Medical and Dental Sciences, University of Birmingham, Birmingham B15 2TT, UK; k.raza@bham.ac.uk duration and favourable outcomes are not linear and that, for all outcomes, a point is reached after which the benefit gained by reducing time to treatment is lessened. Specifically for the authors' primary outcome (DMARD-free sustained remission), the window appeared to start closing at 14.9 weeks for patients in the Leiden cohort and 19.1 weeks in the Evaluation et Suivi de POlyarthrites Indifférenciées Récentes (ESPOIR) cohort. The authors are careful to highlight the relatively wide CIs around these data (the 95\% CIs were 12.3-16.0 weeks in the Leiden cohort and 12.3-28 weeks in the ESPOIR cohort). Nevertheless, at face value, these data do suggest that there is a period, within approximately the first 6 months or potentially an even shorter period, in which DMARD treatment leads to significantly improved long-term outcomes. The authors avoid the easy criticism that DMARD-free remission is a rare outcome and largely restricted to seronegative disease by investigating and identifying a similar relationship for their other chosen outcomes.

These data have important clinical and research implications. From a clinical perspective, accessing patients with new onset RA in a timely fashion can be challenging. European data from 2009 and 2010 showed that the median delay from symptom onset to patients seeing a rheumatologist across 10 centres was 24 weeks. $^{7}$ A significant proportion of patients are thus falling outside the therapeutic window. Delays in the assessment of patients by rheumatologists occur at three main levels: (1) delay on the part of the patient in seeking help in the first instance, for example, in seeing their general practitioner (GP), (2) delay on the part of the GP in referring to a rheumatologist and (3) delay on the part of the rheumatologist in assessing the patient once referred. ${ }^{8-10}$ The extent to which each of these components contributes to the overall delay will be strongly influenced by local healthcare environments but a balanced approach to minimising delays at all three levels is likely to lead to optimum patient outcomes. In countries where patient delay is a significant contributing factor, public health campaigns should address reasons why patients with RA typically delay in seeking help, including the fact that many individuals are unaware of RA, their personal risk of RA or that their symptoms may represent the onset of a disease for which prompt therapy improves long-term outcomes. ${ }^{11}$ The latter is a particular issue when initial symptoms are mild and only progress slowly. ${ }^{11}$ In countries where delay at a primary care level has been identified as a problem, innovative strategies have been developed to speed up access to care. An example whose positive impact has recently been demonstrated involves a package of GP education and the establishment of a rapid access 'Early Arthritis Recognition Clinic' to which GPs can refer patients with equivocal synovitis and where patients are offered short appointments to confirm or exclude synovial inflammation. ${ }^{12}$

The presence of a 'window of opportunity' raises the important question as to the mechanism underlying it. Potential mechanisms include the possibilities that synovitis is qualitatively different within the window (eg, that the cellular and cytokine environment is different between early and late synovitis) and therefore more tractable to therapy. Alternatively, synovitis may be quantitatively different within the window and that once a threshold is reached for the amount of synovium involved treatment is no longer as effective. ${ }^{13}$ Data related to synovial pathology within the first few months after the onset of symptoms compared with longerstanding RA are limited, although some data exist to suggest that disease processes may be different in the earliest stages of disease. ${ }^{14}$ Further research is clearly required in this area, as unique pathological processes operating during the earliest stages of clinically apparent synovitis may suggest that therapeutic approaches should be modified according to whether treatment is being administered within the therapeutic window or not.

While the data presented by van Nies et al strongly support the concept of a time-limited window of opportunity, some alternative explanations should be considered. Patients with RA are likely to be treated early if they present early and treated late if they present late. Previous work from the Leiden group has identified that a gradual onset of symptoms and the involvement of small joints at onset are independently associated with a longer duration of total delay in assessment by a rheumatologist. ${ }^{2}$ If an insidious onset of small joint synovitis characterises a form of RA with a worse natural history, this may explain some of the relationship 
between symptom duration at the time therapy is initiated and clinical outcome. In contrast, if patients with a rapid onset of RA predominantly affecting the larger joints are over-represented within the population treated early, and this form of RA has a better natural history, then the analysis of cohort data such as from the Leiden Early Arthritis Clinic and ESPOIR is likely to suggest the presence of a 'therapeutic window' unless patients are stratified by mode of onset and other variables known to be associated with delays in access to care.

If we do accept that a window of opportunity exists for patients with RA, then the data reported by van Nies et al suggest that it can be timed from the onset of the first patient reported 'musculoskeletal symptom (either pain or swelling)' deemed 'relevant to the current complaint'. Defining the earliest symptoms 'relevant' to RA is a hugely challenging issue. ${ }^{15}$ Patients with new onset RA present with a wide range of symptoms besides joint pain and swelling including fatigue and morning stiffness. ${ }^{16} 17$ If a patient were to present with a 9-month history of fatigue and stiffness and a 3-week history of joint pain and swelling would they be inside or outside the therapeutic window? While the data from van Nies et al suggest that such a patient would fall within the window, future work should address the length of the window in relation to different types of RA onset. The issue of patients presenting with anti-citrullinated peptide antibody (ACPA) positivity and arthralgia $^{18}$ is particularly interesting in this context. Many such patients will develop RA. ${ }^{19}$ The data reported by van Nies et al suggest that if the duration of arthralgia lasts longer than 6 months before RA develops, then, even if the patient is treated as soon as their joints swell, they will have missed the "window of opportunity' because the entirety of their personal 'window of opportunity' will have been used up during their arthralgia phase. Further research, including the systematic use of imaging modalities such as ultrasound and MRI in patients at different stages of disease prior to the development of RA, ${ }^{18}$ should be undertaken in order to better understand the relationship between symptom duration, the true extent of synovitis, prognosis and response to therapy. ${ }^{20-24}$

The paper by van Nies et al adds to an extensive body of literature showing that early treatment of RA is associated with improved outcomes. The data are important because they suggest the presence of a time-limited window of opportunity-a widely accepted concept for which there has actually been relatively limited supporting evidence. As we try to further improve outcomes for patients with RA, it is inevitable that interest will be focused on the earliest phases of disease. As this happens, it is critical that clinical characteristics and disease mechanisms operating during this phase are better understood. In achieving this, we will understand the true clinical correlates of any window of opportunity and be able to offer treatments tailored to pathological processes operating within it.

Contributors This editorial was jointly written by KR and $\mathrm{AF}$.

Competing interests KR has received honoraria from Pfizer, AbbVie and BMS and meeting expenses from Pfizer and Roche.

Provenance and peer review Commissioned; internally peer reviewed.

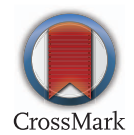

To cite Raza K, Filer A. Ann Rheum Dis 2015;74:793-794.

Received 17 January 2015

Accepted 22 February 2015

Published Online First 10 March 2015

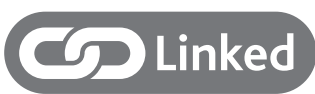

- http://dx.doi.org/10.1136/annrheumdis-2014206047

Ann Rheum Dis 2015:74:793-794.

doi:10.1136/annrheumdis-2014-206993

\section{REFERENCES}

1 Nell VP, Machold KP, Eberl G, et al. Benefit of very early referral and very early therapy with disease-modifying anti-rheumatic drugs in patients with early rheumatoid arthritis. Rheumatology (Oxford) 2004;43:906-14.

2 van der Linden MP, le Cessie S, Raza K, et al. Long-term impact of delay in assessment of patients with early arthritis. Arthritis Rheum 2010;62:3537-46.

3 Kyburz D, Gabay C, Michel BA, et al. The long-term impact of early treatment of rheumatoid arthritis on radiographic progression: a population-based cohort study. Rheumatology (Oxford) 2011;50:1106-10.

4 Finckh $\mathrm{A}$, Liang $\mathrm{MH}$, van Herckenrode $\mathrm{CM}$, et al. Long-term impact of early treatment on radiographic progression in rheumatoid arthritis: a meta-analysis. Arthritis Rheum 2006:55:864-72.

5 Landewe RB, Boers M, Verhoeven AC, et al. COBRA combination therapy in patients with early rheumatoid arthritis: long-term structural benefits of a brief intervention. Arthritis Rheum 2002;46:347-56

6 van Nies JA, Tsonaka R, Gaujoux-Viala C, et al. Evaluating relationships between symptom duration and persistence of rheumatoid arthritis: does a window of opportunity exist? Results on the Leiden Early Arthritis Clinic and ESPOIR cohorts. Ann Rheum Dis 2015:74:806-12.

7 Raza K, Stack R, Kumar K, et al. Delays in assessment of patients with rheumatoid arthritis: variations across Europe. Ann Rheum Dis 2011;70:1822-5.

8 Villeneuve E, Nam JL, Bell MJ, et al. A systematic literature review of strategies promoting early referral and reducing delays in the diagnosis and management of inflammatory arthritis. Ann Rheum Dis 2013;72:13-22.

9 Kumar K, Daley E, Carruthers DM, et al. Delay in presentation to primary care physicians is the main reason why patients with rheumatoid arthritis are seen late by rheumatologists. Rheumatology (Oxford) 2007:46:1438-40.

10 De Cock D, Meyfroidt S, Joly J, et al. A detailed analysis of treatment delay from the onset of symptoms in early rheumatoid arthritis patients. Scand J Rheumatol 2014;43:1-8.

11 Stack RJ, Shaw K, Mallen C, et al. Delays in help seeking at the onset of the symptoms of rheumatoid arthritis: a systematic synthesis of qualitative literature. Ann Rheum Dis 2012;71:493-7.

12 van Nies JA, Brouwer E, van Gaalen FA, et al. Improved early identification of arthritis: evaluating the efficacy of Early Arthritis Recognition Clinics. Ann Rheum Dis 2013;72:1295-301.

13 Raza K. The Michael Mason prize: early rheumatoid arthritis--the window narrows. Rheumatology (Oxford) 2010;49:406-10.

14 Raza K, Falciani F, Curnow SJ, et al. Early rheumatoid arthritis is characterized by a distinct and transient synovial fluid cytokine profile of T cell and stromal cell origin. Arthritis Res Ther 2005;7:R784-95.

15 Raza K, Saber TP, Kvien TK, et al. Timing the therapeutic window of opportunity in early rheumatoid arthritis: proposal for definitions of disease duration in clinical trials. Ann Rheum Dis 2012;71:1921-3.

16 Stack RJ, Sahni M, Mallen CD, et al. Symptom complexes at the earliest phases of rheumatoid arthritis: a synthesis of the qualitative literature. Arthritis Care Res (Hoboken) 2013;65:1916-26.

17 Stack RJ, van Tuyl LH, Sloots M, et al. Symptom complexes in patients with seropositive arthralgia and in patients newly diagnosed with rheumatoid arthritis: a qualitative exploration of symptom development. Rheumatology (Oxford) 2014;53:1646-53.

18 Gerlag DM, Raza K, van Baarsen LG, et al. EULAR recommendations for terminology and research in individuals at risk of rheumatoid arthritis: report from the Study Group for Risk Factors for Rheumatoid Arthritis. Ann Rheum Dis 2012;71:638-41.

19 van de Stadt LA, Witte BI, Bos WH, et al. A prediction rule for the development of arthritis in seropositive arthralgia patients. Ann Rheum Dis 2013;72:1920-6.

20 van de Stadt $L A$, Bos WH, Meursinge Reynders $M$, et al. The value of ultrasonography in predicting arthritis in auto-antibody positive arthralgia patients: a prospective cohort study. Arthritis Res Ther 2010;12:R98.

21 Rakieh C, Nam JL, Hunt L, et al. Predicting the development of clinical arthritis in anti-CCP positive individuals with non-specific musculoskeletal symptoms: a prospective observational cohort study. Ann Rheum Dis 2014. Published Online First: $12 \mathrm{Apr}$ 2014. doi:10.1136/annrheumdis-2014-205227

22 Filer $A$, de Pablo $P$, Allen $G$, et al. Utility of ultrasound joint counts in the prediction of rheumatoid arthritis in patients with very early synovitis. Ann Rheum Dis 2011;70:500-7.

23 Stomp W, Krabben A, van der Heijde D, et al. Are rheumatoid arthritis patients discernible from other early arthritis patients using $1.5 \mathrm{~T}$ extremity magnetic resonance imaging? A large cross-sectional study. J Rheumatol 2014;41:1630-7.

24 Krabben A, Stomp W, van Nies JA, et al. MRI-detected subclinical joint inflammation is associated with radiographic progression. Ann Rheum Dis 2014;73:2034-7. 